\title{
The acquired immunodeficiency syndrome in the State of Rio de Janeiro, Brazil: a spatio-temporal analysis of cases reported in the period 2001-2010
}

\author{
André T. J. Alves ${ }^{1}$, Flávio F. Nobre ${ }^{1}$ \\ ${ }^{1}$ Biomedical Engineering Program, Federal University of Rio de Janeiro, Rio de Janeiro, Brazil
}

\begin{abstract}
Despite increased funding for research on the human immunodeficiency virus (HIV) and the acquired immunodeficiency syndrome (AIDS), neither vaccine nor cure is yet in sight. Surveillance and prevention are essential for disease intervention, and it is recognised that spatio-temporal analysis of AIDS cases can assist the decision-making process for control of the disease. This study investigated the dynamic, spatial distribution of notified AIDS cases in the State of Rio de Janeiro, Brazil, between 2001 and 2010, based on the annual incidence in each municipality. Sequential choropleth maps were developed and used to analyse the incidence distribution and Moran's I spatial autocorrelation statistics was applied for characterisation of the spatio-temporal distribution pattern. A significant, positive spatial autocorrelation of AIDS incidence was observed indicating that municipalities with high incidence are likely to be close to other municipalities with similarly high incidence and, conversely, municipalities with low incidence are likely to be surrounded by municipalities with low incidence. Two clusters were identified; one hotspot related to the State Capital and the other with low to intermediate AIDS incidence comprising municipalities in the north-eastern region of the State of Rio de Janeiro.
\end{abstract}

Keywords: AIDS incidence, spatial analysis, disease mapping, cluster, Brazil.

\section{Introduction}

The very first cases of acquired immunodeficiency syndrome (AIDS) were reported in 1981 by the Centers for Disease Control and Prevention (CDC, 1981). Since then, more than 65 million people have been infected with the human immunodeficiency virus (HIV) (UNAIDS, 2012). The estimate in 2010 was that more than 34 million people worldwide were infected with HIV with an annual addition of 2.7 million new infections and 1.8 million AIDS-related deaths (UNAIDS, 2011).

In Brazil, the epidemic is followed by the "Programa Nacional de Doenças Sexualmente Transmissíveis/AIDS" (MS). The first cases were detected in the cities of São Paulo and Rio de Janeiro in the early 1980s (Castilho et al., 1994), and most new cases continued to be reported from there for some time. Only after 1992 the infection spread to all five regions of the country, but the Southeast still accounts for the largest fraction $(41.8 \%$ in 2012) of reported cases (Bastos and

Corresponding author:

André T. J. Alves

Biomedical Engineering Program

Federal University of Rio de Janeiro

Av. Horácio Macedo 2030, Rio de Janeiro, Brazil

Tel. +55 21 2562-8624; Fax +55 21 2562-8591

E-mail: andrethiago@peb.ufrj.br
Barcellos, 1995; MS, 2013). From 1980 to mid-2013, there were 686,478 new cases of AIDS within the States of Rio de Janeiro and São Paulo accounting for almost half that number (47\%). In 2010, an additional 34,218 cases were diagnosed with 11,081 from these two states, a figure corresponding to about $30 \%$ of all cases reported that year.

The initial, strong increase in Brazil rapidly resulted in the country accounting for approximately one-third of all cases reported in Latin America, but the AIDS occurrence then stabilised and have continued to do so during the last 10 years with a particular reduction of new cases in the Southeast (UNAIDS, 2010; MS, 2011). In spite of this reduction, however, the incidence of new AIDS cases is still high in Rio de Janeiro, which has the fourth highest AIDS incidence among the Brazilian states and the highest incidence in the Southeast (MS, 2013).

Increased research funding notwithstanding, there is currently no vaccine or cure for HIV/AIDS. The use of highly active antiretroviral therapy (HAART) in the treatment of individuals infected with HIV has reduced the mortality risk and AIDS-related complications. Since 1996, Brazil has provided universal and free access to HAART (Dourado et al., 2006). The country is widely recognised as a leader in the fight against AIDS in low- and middle-income countries (Biehl, 2006). In 2002, half of all people in the world on antiretroviral treatment lived in Brazil, which 
reached the $80 \%$ coverage target in 2011 (UNAIDS, 2013). Despite the success with the HAART programme, there are some specific challenges to maintain universal access to treatment policy, such as the increased number of patients on treatment and the increased complexity and costs of providing antiretroviral drugs (Greco and Simão, 2007). Given these difficulties in maintaining the HAART approach, prevention is still essential for achieving better control of the epidemic. In addition, the severity of AIDS and the extreme social and personal implications of identifying HIV-infected persons make surveillance an invaluable tool.

Public health surveillance, defined as the ongoing systematic collection, analysis and dissemination of data relevant to prevention or control (Thacker et al., 1989), is essential for our understanding of epidemiological patterns and scopes of communicable diseases, and AIDS cases and HIV infections are no exceptions (Chin, 1990). Case surveillance data provide the basis for assessing burdens of disease and are used to guide public health action at the federal, state and local levels. Knowledge of the number of people diagnosed with AIDS each year and their stage of disease when diagnosed is as important for planning and resource allocation as for monitoring of trends and disparities between groups (CDC, 2012). Improved surveillance information should help public health planners establish more rational priorities, which should, in turn, lead to earlier detection and greater control of epidemics (Thacker et al., 1989).

Since the 1990s, spatial, analytical methods and geographical information systems (GIS) have been increasingly applied in ecological studies as a tool for analysis and visualization of health-related spatial data in epidemiological research (Moore and Carpenter, 1999; Robinson, 2000). Spatial analysis can also assist decision-making for disease intervention and has been employed for surveillance purposes in the field of public health. Several studies have focused on investigating the geographical distribution, spatial clusters and spread of AIDS describing the dynamic trends using spatio-temporal analysis or statistical methods (Berkley et al., 1989; Lam et al., 1996; Hixson et al., 2011; Peng et al., 2011; Manda et al., 2012). Spatial clustering analysis provides useful information on where to intervene and can also contribute to more effective intervention. Knowing what to do, and where to do it, provide the key ingredients for effective action against AIDS in communities that are severely affected by this epidemic (Karim, 2009).

In the present study, we analysed the spatial distri- bution of AIDS cases in the State of Rio de Janeiro. Based on exploratory spatial data analysis methods, the annual incidence of AIDS by municipality for the period 2001-2010 and Moran's I spatial autocorrelation statistic, we examined whether the epidemic spreads in a recognisable manner and whether clusters with a similar incidence of AIDS exist.

\section{Materials and methods}

\section{Data source}

Since the 1980s, the epidemiological surveillance of HIV/AIDS in Brazil is based on compulsory notification of AIDS cases through the Notifiable Diseases Information System (SINAN) and all Brazilian municipalities are using standardised instruments for the notification of cases since 2003. Our study included all AIDS cases notified to the Brazilian Ministry of Health by the 92 municipalities of the State of Rio de Janeiro during the 2001-2010 period. Data were obtained from SINAN according to the year of diagnosis and patient place of residence. Information about the annual resident populations of the municipalities was obtained from the projections provided by the Brazilian Institute of Geography and Statistics (IBGE). A digital map of the State of Rio de Janeiro with the boundaries of all municipalities was also obtained from IBGE.

\section{Estimating AIDS incidence}

Estimates of the annual AIDS incidence for the 2001-2010 period were calculated based on the 3-year moving average. These estimates were used to reduce the effect that can occur as the result of underreporting and also to prevent biases in the estimated AIDS incidence, which can be affected by small number of residents in some egions. The 3-year moving-average incidence rates for each municipality were calculated using the total number of AIDS cases reported for the 3 -year period divided by three and then divided by the estimated mid-year population of the municipality in question, resulting in a total of eight estimated rates, which were analysed using two approaches. The first is based on sequential choropleth maps of the estimated incidences, while the second consists of spatial correlation analysis. The $\mathrm{R}$ statistical software, version 2.14.2 (R Development Core Team, 2012), with the Maptools (Lewin-Koh and Bivand, 2012) and Spatstat (Baddeley and Turner, 2005) packages, was used for all analyses and also for map generation. 


\section{Spatial analysis}

To examine change over time, the incidence of AIDS per 100,000 was divided into four classes [0-13.32], [13.32-22.26], [22.26-30.02] and [>30.02], which were used to obtain eight choropleth maps. This was done using the natural breaks method that finds a partition with the least difference between the samples in the same class and maximises the difference between classes (Jenks and Caspall, 1971). It is a data-specific classification that we used to visualize and facilitate comparison between the eight time periods by defining the same four class intervals using the averaged incidence for each municipality for the 2001-2010 period.

The Moran's I spatial autocorrelation statistic was used to evaluate if the similarity in AIDS incidence between nearby areas was greater than would be expected from a random pattern (Bailey and Gatrell, 1995). The Moran coefficient ranges from -1 to +1 and can be interpreted as follows: a value close to 0 indicates spatial randomness, whereas a positive value indicates positive autocorrelation and a negative value indicates negative autocorrelation. It allows characterization of the spatial dependence of AIDS incidence rates. The global Moran coefficient is based on the cross-products of the deviations from the mean and is calculated for $n$ observations as follows:

$$
I=\frac{\sum_{i}^{n} \sum_{i}^{n} W_{i j} Z_{i} Z_{j}}{\sum_{i}^{n} Z_{i}^{2}}
$$

(equation 1)

where $n$ is the number of areas; $Z_{i}$ the difference between the value of the incidence rate at location $i$ and the average of all rates; $Z_{j}$ the difference between the value of the incidence rates of the neighbours at location $i$ and the average of all rates; $W_{i j}$ the row standardised weights assigned according to the topological relationship between locations $i$ and $j$, where $W_{i j}=0$ if $i$ and $j$ are not neighbours.

Moran's I provides a measure of overall clustering but does not indicate the location of clusters or outliers. In order to assess local spatial clustering, it is more useful to have a measure of association for each unit (the local Moran's I) also known as the local index of spatial autocorrelation (LISA), which is calculated as follows:

$$
I_{i}=\frac{Z_{i} W_{z}}{\sum_{i}^{n} Z_{i}^{2}}
$$

(equation 2) where

$$
W_{z}=\mid \sum_{j}^{n} W_{i j} Z_{j}
$$

LISA can be analysed using the Moran scatterplot (Fig. 1), where deviations $(\mathrm{Z})$ of the incidence rate in relation to the mean are plotted on the $x$-axis and the weighted average of deviations from the neighbours defined by the matrix $\mathrm{W}_{z}$ on the $y$-axis. This representation shows the linear relationship between $\mathrm{Z}$ and $\mathrm{W}_{z}$, where the slope of the regression line defines the global Moran's I (Anselin, 1996).

The Moran map builds on the Moran scatterplot (which helps identifying the nature of the spatial autocorrelation between municipalities) and incorporates information on the significance $(\mathrm{P}<0.05)$ of local spatial patterns. Analysis of the scatterplot identifies four types of association: quadrant Q1 (high-high) has regions with a high incidence rate that have neighbouring regions with a high incidence rate; Q2 (lowlow) has neighbouring regions with low rates; while Q3 (high-low) and Q4 (low-high) have regions in which the value of the measured attribute is not similar to that of their neighbours, showing the presence of a negative association between the regions, i.e. areas with a high (low) incidence have neighbours with a low (high) incidence. The areas located in these two quadrants can be regarded as transition regions (Anselin, 1995).

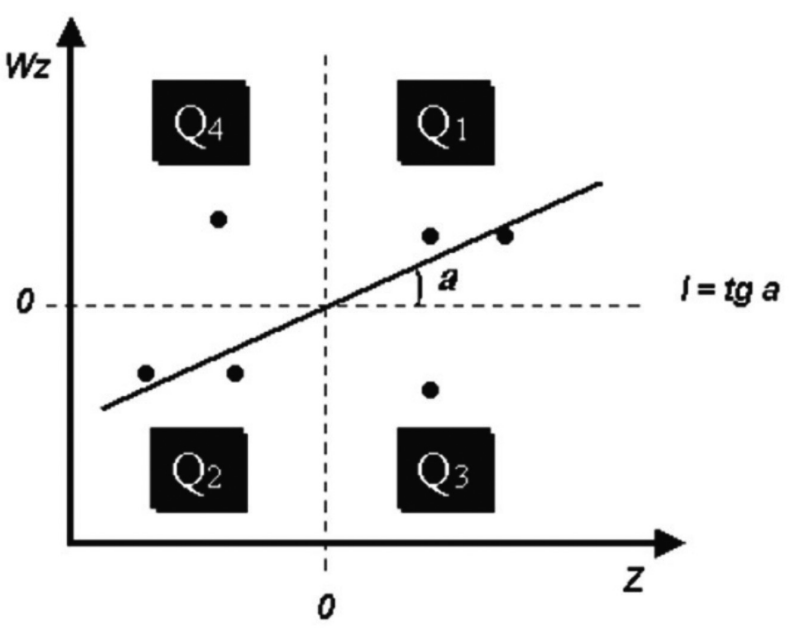

Fig. 1. The Moran scatterplot with the slope of the regression line defining the global Moran's $I$. The linear relationship between $\mathrm{Z}$ (incidence rate in relation to the mean) and $W_{z}$ (the weighted average of deviations from the neighbours) where Q1 represents the high-high; Q2 the low-low; Q3 the high-low; and Q4 the low-high relationship. 

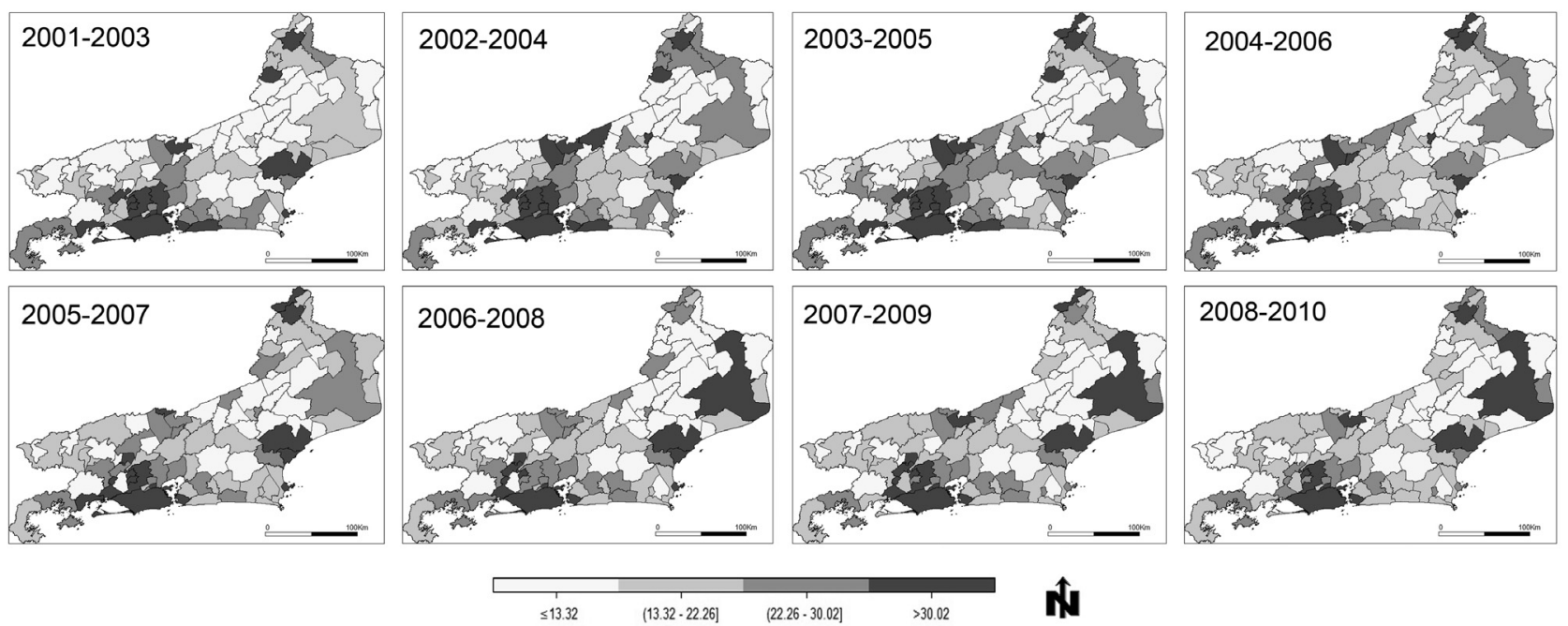

Fig. 2. Sequential choropleth maps of the AIDS incidence per 100,000 in the State of Rio de Janeiro, 2001-2010. Incidence divided into four classes based on the natural breaks method.

\section{Results}

The sequential choropleth maps show the AIDS incidence in each municipality for each period. Visual analysis of the spatial distribution of this incidence shows no evidence of spatial spread over the studied period, but rather, the predominance of a clustering process with respect to the occurrence of AIDS. Fig. 2 shows these maps and the spatial distribution of the 3year moving average of AIDS incidence rates per 100,000 for each municipality with reference made to the four map classes obtained by the natural breaks method. The municipalities with the highest incidences are around the State Capital, at the bottom left of each subfigure. Municipalities with intermediate to high incidence values during the study period are seen at the top right of each subfigure. Low AIDS incidence areas can be seen across the state and are mostly seen in smaller municipalities with small populations.

Table 1. Statistical significance of the AIDS incidence in the 92 municipalities of the State of Rio de Janeiro, 2001-2010.

\begin{tabular}{lcc}
\hline Period & Global Moran's $I$ & P-value \\
\hline $2001-2003$ & 0.304 & $<0.001$ \\
$2002-2004$ & 0.208 & 0.002 \\
$2003-2005$ & 0.222 & $<0.001$ \\
$2004-2006$ & 0.175 & 0.007 \\
$2005-2007$ & 0.254 & $<0.001$ \\
$2006-2008$ & 0.251 & $<0.001$ \\
$2007-2009$ & 0.281 & $<0.001$ \\
$2008-2010$ & 0.314 & 0.005 \\
\hline
\end{tabular}

Global Moran's I spatial autocorrelation statistics were calculated for each of the eight periods (Table 1). A significant positive spatial autocorrelation of AIDS incidence was found for all periods, indicating that high-incidence municipalities are likely to be close to other high-incidence municipalities with and, conversely, municipalities characterised by low incidence are likely to be close to other municipalities with a low incidence. Spatial autocorrelation varied over the study period, reaching its nadir during 2004-2006 and its peak during 2008-2010, the last period of analysis. Since the global Moran's I autocorrelation statistic does not indicate the location of spatial clusters, clusters of high rates, of low rates and regions with intermediate rates were identified by means of LISA. Statistically significant values were plotted on the Moran maps for the eight different periods. Fig. 3 shows four types of spatial correlation (high-high, low-low, high-low and low-high). The Moran maps show two clusters, one of high AIDS incidence located around the State Capital and one of low to intermediate incidence comprising municipalities located predominantly in the north-eastern region of the State of Rio de Janeiro.

Municipalities from the metropolitan region of Rio de Janeiro belong to a high-high cluster. Over the years, there was a slight reduction in the number of municipalities belonging to this cluster, coinciding with municipalities around the metropolitan region changing from a high-high pattern to a low-high pattern. This is a common trend characteristic of municipalities with a low incidence of the disease located next to high-incidence areas. 

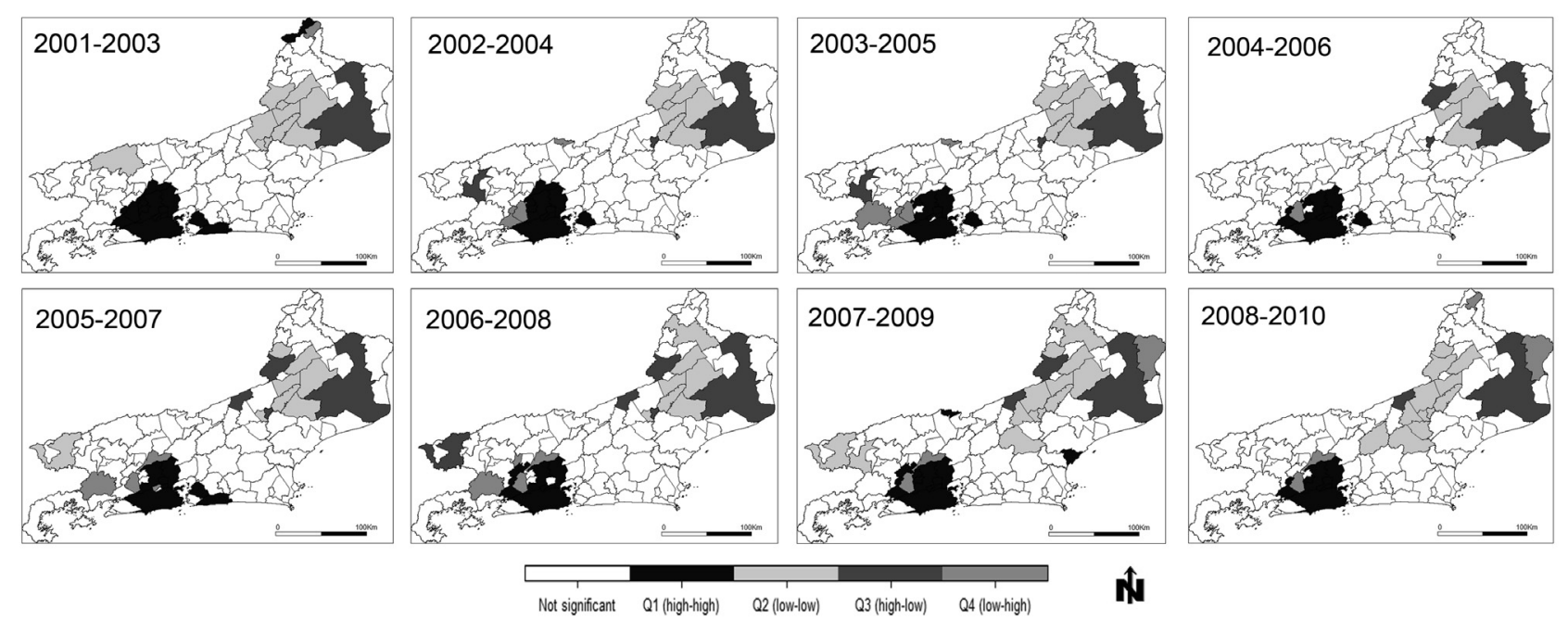

Fig. 3. Moran map of the AIDS incidence in the State of Rio de Janeiro, 2001-2010. The legend represents the four quadrants of the Moran scatter plot with the municipalities shown in white being without statistically significant autocorrelation.

There is a cluster of municipalities in the north-eastern region of the state with a predominantly low-low pattern of incidence. In this region, some municipalities shifted category from low-low to high-low over time, displaying a more dynamic spatial pattern. However, it should be underlined that Campos dos Goytacazes, which is located in this region and is the municipality with the largest land area in the state, exhibited the high-low type of spatial correlation for all eight periods. As can be seen in Fig. 3, most of the municipalities, particularly those located in the centre of the state showed no statistically significant autocorrelation.

\section{Discussion}

Visual inspection of sequential choropleth maps is commonly used by health programmes to identify priority areas for intervention, but this approach is limited, since it does not consider the influence of neighbouring municipalities on the incidence pattern. The choropleth maps used here show the AIDS incidence in each municipality for each period, which enables visualization of the spatial distribution of AIDS incidence and of how the epidemic spread throughout the municipalities of the State of Rio de Janeiro during the study period. However, limitations regarding the choice of classification process for the incidence rate, which can be based on quintiles or ad-hoc methods, need to be considered. The spatial approach employed in this study can be used to identify and monitor highincidence areas over different periods of time. Knowledge of the spatial dynamics of AIDS occur- rence may allow improvement of surveillance actions by taking into account the specificities of municipalities and their neighbours. Almost 30 years after the first cases of AIDS were reported in the municipality of Rio de Janeiro, the Moran map shows that there is still a cluster of high AIDS incidence comprising the metropolitan region of the State of Rio de Janeiro.

In contrast to the high-incidence profile of the major urban centres of the state, much of the low incidence was seen in smaller municipalities. A cluster of low-low incidence was found in the north-eastern part of the state, which comprises municipalities with small populations. This region is the farthest from the locus of high AIDS incidence surrounding the State Capital; nevertheless, some neighbouring municipalities of this low-low cluster show a high incidence, a condition that characterises the high-low pattern depicted in the spatial correlation. Campos dos Goytacazes, the most populous municipality outside the metropolitan region and an important urban centre of upstate Rio de Janeiro, is a good example of this. Due to its proximity to municipalities with a low AIDS incidence and the possibility of spread, this municipality must be seen as a critical area requiring special intervention.

Geographical clustering of new HIV infections is a common phenomenon, particularly in urban areas (Shacham et al., 2013). The high AIDS incidence, which was observed in the metropolitan region of the State of Rio de Janeiro, can also be seen in other metropolitan areas, such as in some populated urban regions of South Africa (Manda et al., 2012), the metropolitan area of Atlanta (Hixson, 2011) and another 
11 large urban areas in the United States of America (Hall et al., 2010). In 2011, the AIDS incidence in Brazil was 20.2 cases per 100,000. Much of the municipalities of Rio de Janeiro, especially those from the metropolitan region, had a higher incidence than that observed at the national level during the whole study period. Indeed, the incidence is still high around the State Capital despite all public health policies that has made Brazil's response to AIDS internationally acknowledged.

Difficulties accessing antiretroviral treatment have been identified in Rio's metropolitan area, e.g. in settings such as shanty towns (favelas), where there is concern around the free access to, and availability of, antiretroviral treatment as well as the sustainability of public health policies (Cataldo, 2008). In addition, the access to the full package of interventions for the prevention of HIV vertical transmission is still low in the metropolitan region of the state (da Silva Pires Araujo et al., 2014). Significant proportions of pregnant women living with HIV either remain undiagnosed or do not start on antiretrovirals medicines even when diagnosed. The State Health Department in Rio de Janeiro (Secretaria de Estado de Saúde - SES/RJ) estimates that $30 \%$ of all pregnant women with AIDS in the state have not used antiretrovirals at childbirth, which could prevent vertical contamination, and 24\% only discovered they were HIV-positive during pregnancy (SES/RJ, 2013).

Late diagnosis is a great concern in Rio de Janeiro. In 2012, according to the State Department of Health, $27.5 \%$ of patients in the state were diagnosed late (SES/RJ, 2013), which means that in that year alone about $28 \%$ of HIV-infected persons did not receive HAART since they were unaware of the infection. Late diagnosis has consequences for AIDS incidence as well since people unaware of their HIV status contribute to continuing HIV transmission. Furthermore, both the National Collaborating Centre for Infectious Diseases (NCCID) and individual scientists agree that early diagnosis would lead to more rapid HAART support and reduced spread of the disease by lowering the viral load both in blood plasma and the genital tract (Porco et al., 2004; NCCID, 2010).

The Metropolitan Rio de Janeiro area has hundreds of low-income communities and some studies indicate that higher AIDS incidence tend to occur in poorer neighbourhoods (Greco and Simão, 2007; Hixson et al., 2011; Shacham et al., 2013; da Silva Pires Araujo et al., 2014). It has been suggested that poverty may increase the vulnerability to HIV infection since it hinders access to information, preventive measures and healthcare. In fact, a large disparity still exists in the metropolitan region of the State of Rio de Janeiro; low-income communities are characterised by significant levels of social inequality and millions are marginalised. In order to tackle these problems related to the high AIDS incidence in some municipalities of Rio de Janeiro, new policies are required for a better integration of prevention and treatment. For 2014, new programmes are planned in the State of Rio de Janeiro to improve prevention of AIDS, early diagnosis and HAART access (SES/RJ, 2013). In this sense, the clusters of high AIDS incidence outlined in this study should be seen as critical areas, where surveillance efforts should be concentrated and public health interventions are most required.

\section{Acknowledgements}

The research was funded by a research grant from the Brazilian National Council for Scientific and Technological Development (CNPq). A.T.J. Alves acknowledges the scholarship and support received from the Coordination for the Improvement of Higher Level Personnel (Capes).

\section{References}

Anselin L, 1995. Local indicators of spatial association-LISA. Geogr Anal 27, 93-115.

Anselin L, 1996. The Moran scatterplot as an ESDA tool to assess local instability in spatial association. In: Spatial analytical perspectives on GIS. Fischer M, Scholten H, Unwin D (eds). London: Taylor and Francis, 111-125 pp.

Baddeley A, Turner R, 2005. Spatstat: an R package for analyzing spatial point patterns. J Stat Softw 12, 1-42.

Bailey TC, Gatrell AC, 1995. Interactive spatial data analysis. New York: Longman Scientific \& Technical, 413 pp.

Bastos FI, Barcellos C, 1995. Geografia social da AIDS no Brasil. Rev Saude Publica 29, 52-62.

Berkley S, Okware S, Naamara W, 1989. Surveillance for AIDS in Uganda. AIDS 3, 79-85.

Biehl J, 2006. Pharmaceutical governance. In: Global pharmaceuticals. Petryna A, Lakoff A, Kleinman A (eds). Ethics, Markets, Practices. Durham: Duke University Press, 206-239 pp.

Castilho EA, Chequer P, Struchiner C, 1994. A epidemiologia da AIDS no Brasil. In: A Aids no Brasil (1982-1992). Parker R, Bastos C, Galvão J, Pedrosa JS, (eds). Rio de Janeiro: Relume-Dumará, 59-67 pp.

Cataldo F, 2008. New forms of citizenship and socio-political inclusion: accessing antiretroviral therapy in a Rio de Janeiro favela. Sociol Health Illn 30, 900-912.

CDC, 1981. Pneumocystis pneumonia - Los Angeles. Morb Mortal Wkly Rep 30, 250-252. 
CDC, 2012. HIV surveillance supported by the Division of HIV/AIDS Prevention. Atlanta, Georgia: Centers for Disease Control and Prevention. Available at: http://www.cdc.gov/ hiv/statistics/recommendations/publications.html. (accessed on April 2014).

Chin J, 1990. Public health surveillance of AIDS and HIV infections. Bull World Health Organ 68, 529-536.

da Silva Pires Araujo E, Friedman RK, Camacho LAB, Derrico M, Moreira RI, Calvet GA, de Oliveira MS, Veloso VG, Pilotto JH, Grinsztejn B, 2014. Cascade of access to interventions to prevent HIV mother to child transmission in the metropolitan area of Rio de Janeiro, Brazil. Braz J Infect Dis 1-9.

Dourado I, Veras MASM, Barreira D, Brito AM, 2006. AIDS epidemic trends after the introduction of antiretroviral therapy in Brazil. Rev Saude Publica 40, 9-17.

Greco DB, Simão M, 2007. Brazilian policy of universal access to AIDS treatment: sustainability challenges and perspectives. AIDS 21, S37-S45.

Hall HI, Espinoza L, Benbow N, Hu YW, for the Urban Areas HIV Surveillance Workgroup, 2010. Epidemiology of HIV infection in large urban areas in the United States. PLoS One 5, e12756.

Hixson BA, Omer SB, del Rio C, Frew PM, 2011. Spatial clustering of HIV prevalence in Atlanta, Georgia and population characteristics associated with case concentrations. J Urban Health 88, 129-141.

Jenks GF, Caspall FC, 1971. Error on choroplethic maps: definition, measurement, reduction. Ann Assoc Am Geogr 61, 217-244.

Karim SSA, 2009. Spatial clustering of HIV infection: providing clues for effective HIV prevention. Int J Epidemiol 38, 1016 1017.

Lam NS-N, Fan M, Liu K, 1996. Spatial-temporal spread of the AIDS epidemic, 1982-1990: a correlogram analysis of four regions of the United States. Geogr Anal 28, 93-107.

Lewin-Koh NJ, Bivand R, 2012. Maptools: tools for reading and handling spatial objects. Available at: http://CRAN.Rproject.org/package=maptools (accessed on April 2014).

Manda SOM, Lombard CJ, Mosala T, 2012. Divergent spatial patterns in the prevalence of the human immunodeficiency virus (HIV) and syphilis in South African pregnant women. Geospat Health 6, 221-231.

Moore DA, Carpenter TE, 1999. Spatial analytical methods and geographic information systems: use in health research and epidemiology. Epidemiol Rev 21, 143-161.

MS, 2011. Ministério da Saúde - Secretaria de Vigilância em Saúde - Departamento de DST, Aids e Hepatites Virais. Bol Epidemiol AIDS/DST, pp. 1-159.

MS, 2013. Ministério da Saúde - Secretaria de Vigilância em Saúde - Departamento de DST, Aids e Hepatites Virais. Bol Epidemiol AIDS/DST, pp. 1-64.

NCCID, 2010. Interventions to prevent HIV transmission in serodiscordant couples. Winnipeg, Manitoba: National Collaborating Centre for Infectious Diseases. Available at: http://www.nccid.ca/files/serodiscordant_couples.pdf. (accessed on March 2014).

Peng ZH, Cheng YJ, Reilly KH, Wang L, Qin QQ, Ding ZW, Ding GW, Ding KQ, Yu RB, Chen F et al., 2011. Spatial distribution of HIV/AIDS in Yunnan province, People's Republic of China. Geospat Health 5, 177-182.

Porco TC, Martin JN, Page-Shafer KA, Cheng A, Charlebois E, Grant RM, Osmond DH, 2004. Decline in HIV infectivity following the introduction of highly active antiretroviral therapy. AIDS 18, 81-88.

R Development Core Team, 2012. R: a language and environment for statistical computing. Vienna: R Foundation for Statistical Computing.

Robinson TP, 2000. Spatial statistics and geographical information systems in epidemiology and public health. Adv Parasitol 47, 81-128.

SES/RJ, 2013. AIDS: Região Metropolitana do RJ concentra maior taxa de incidência da doença. Available at: http://www.saude.rj.gov.br/imprensa-noticias/20411-aidsregiao-metropolitana-do-rj-concentra-maior-taxa-de-incidencia-da-doenca.html (accessed on March 2014).

Shacham E, Lian M, Önen NF, Donovan M, Overton ET, 2013. Are neighborhood conditions associated with HIV management? HIV Med 14, 624-632.

Thacker SB, Berkelman RL, Stroup DF, 1989. The science of public health surveillance. J Public Health Policy 10, 187-203. UNAIDS, 2010. Global report, pp. 1-359.

UNAIDS, 2011. UNAIDS world AIDS day report, pp. 1-48.

UNAIDS, 2012. UNAIDS report on the global AIDS epidemic, pp. 1-106.

UNAIDS, 2013. Global update on HIV treatment 2013: results, impact, and opportunities, pp. 1-124. 